\title{
Analysis on Age Invariance Face Recognition Study and Effects of Intrinsic and Extrinsic Factors on Skin Ageing
}

\author{
Ashutosh Dhamija \\ ECE department, SRM University, Sonepat, \\ Haryana, India
}

\author{
R. B. Dubey \\ EEE department, SRM University, Sonepat, \\ Haryana, India
}

\begin{abstract}
Currently, age invariance face recognition is an emerging research topic and has many potential applications. Face recognition under different intra-individual varieties, for example, demeanors, posture and impediment has been given satisfactory consideration in examination documented. In any case, age invariance confront acknowledgment still faces numerous difficulties because of age related natural changes in nearness of other appearance varieties. This paper studies noticeable distributed literary works to break down and outline work done as such far on age invariant face acknowledgment and to assess them on different scales like computational speed, precision, execution consistency in inborn outward conditions on skin maturing.
\end{abstract}

\section{Keywords}

Age invariance, PC vision, outward conditions, characteristic conditions, maturing databases, ageing skin, intrinsic and extrinsic factors in skin ageing.

\section{INTRODUCTION}

Face acknowledgment is a developing examination point with various potential applications. It is a biometric approach that utilizes robotized techniques to confirm personality of an individual dependent on physiological qualities maturing of an individual realizes an adjustment fit as a fiddle surface of face. It is an exceptionally mind boggling process which relies upon numerous elements like quality example, way of life, stretch, natural conditions and so on. Programmed confront acknowledgment is a vital yet difficult assignment because of maturing varieties, intra-client varieties, for example, present, light, articulation and between client similarity[1].

Age invariant face acknowledgment frameworks were not broadly considered before on account of absence of reasonable databases, yet ongoing coming of FGNET, MORPH and different databases have made this zone accessible for wide research field. $\mathrm{N}$.

Ramanathan and R. Chellappa [2] displayed a Bayesian agecontrast classifier that distinguishes age division between a couple of face pictures of a person. This strategy was appropriate to deal with age movement in grown-up pictures, yet not viable for taking care of age movement in face pictures of youngsters. H Ling and S Soatto et al [3] proposed a hearty face descriptor, slope introduction pyramid, for face confirmation undertakings crosswise over ages. Contrasted with recently utilized descriptors, for example, picture power, new descriptor is progressively strong performs well on face pictures with expansive age contrasts.

N. R. Syambas and U H Purwanto [4] concentrated on advancement of picture pre-handling factors like complexity, brilliance, sharpness in acknowledgment framework for enhanced acknowledgment precision. G Mahalingam and C
Kambhamettu [5] introduced a diagram based picture portrayal and a maturing model built utilizing GMM for every person to show their age varieties basically fit as a fiddle and surface. Here, a two phase approach for acknowledgment is utilized in which a basic deterministic calculation that misuses topology of charts is proposed for productive diagram coordinating between test picture and exhibition picture.

J. S. Nayak and Indiramma M. [6] utilized a self-PCA based strategy to represent peculiarity of impacts of maturing of an individual for age invariant face acknowledgment. Locale around eyes is utilized as input feature highlight rather than whole face as it is progressively steady piece of face. J. S. Nayak and Nagarathna N. et al [7] proposed this self-PCA based face acknowledgment technique to think about maturing impacts by building subspace at individual dimension. Z. Li and U. Park et al [8] utilized a discriminative model to address confront coordinating with insight of age variety. Scale invariant element change (SIFT) and multiscale nearby parallel examples (MLBP) fill in as neighborhood descriptors. Since both SIFT-based nearby highlights and MLBP-based neighborhood highlights range a high-dimensional element space, to keep away from over fitting issue, build up a calculation, called multi-include discriminant investigation (MFDA) to process these two neighborhood highlight spaces in a brought together structure. On facial maturing, age movement and age estimation strategies are studied in $[9,11,12]$. Though, a couple of face verification explores crosswise over maturing alongside age movement and age estimation is accounted for in written works. Introductory deals with impact of maturing on execution of AIFR has been tended to however it just uses FG-NET database [9-13].inspiration for this paper is to give an audit of current age invariant models accessible in writing and gauge best one that satisfies all highlights and conditions.

Rest of this paper is as follows. In Section 2, various aging databases are described. Section 3 deals with age invariance face recognition methods. A near investigation of age invariant face acknowledgment strategies are classified in Section 4. Impacts of natural and extraneous factors on skin maturing are examined in Section 5. Barely any rules for future innovative work are proposed in Section 6. At last, finishing up comments are given in Section 7.

\section{AGING DATABASES}

Databases play very important and crucial role for testing of face recognition algorithms. Amongst many available databases only few of them are address aging problem. Due to lack of appropriate aging databases, age invariant issue has gained its attention very late. Now, many databases are available; a few of them are given below [15]: 


\subsection{FERET}

This contains little age variations. It has total number of 14,126 images which has 1199 subjects \& 365 duplicate set of images. It is hardly used for testing of AIFR algorithms.

\subsection{FG-NET}

It contains 1002 images of 82 different subjects. Its major limitations are less number of subjects available in database.

\subsection{MORPH}

It is a very large database. It has two sets, namely, album $1 \&$ 2. Album 1 covers 1690 face images of 625 subjects in age group of 15-60 years. Album 2 contains 78,207 images of 20,569 different subjects.

\subsection{Cross-age celebrity database (CACD)}

It covers 163,446 images of 2000 subjects. These images are collected from Google Image Search using celebrity name and year as keywords. Meta data details like name, age, identity, birth year is provided additionally.

\subsection{Pinellas Country Sheriff's Office Longitudinal Study (PCSO-LS)}

It contains 1.5 million pictures which are gathered from18, 007 hoodlums captured by PCSO. It subjects have no less than 5 confront pictures gathered over somewhere around 5 years time of range. It doesn't contain pictures of subjects between age gatherings of 0-15 years.

\subsection{Wholsit (WIT)}

It covers 1109 images of 110 subjects which are collected from internet.

\subsection{FACES}

It contains 1026 pictures of 171 subjects with six articulations like impartial, miserable, appall, fear, furious and cheerful. All pictures are frontal with settled light image gathering of $19-80$

\subsection{ADIENCE}

This database is accumulation of wild face pictures. It contains 26,580 pictures of 2284 subjects. This has variety, for example, appearance, commotion, postures and lighting.

\section{AGE INVARIANCE FACE RECOGNITION METHODS}

Facial maturing process influences appearance, shape and surface of human face. Age related changes are reliant on inborn just as extraneous components like, condition, way of life, introduction to sun, push, and infections and so on. Distinctive maturing design saw amid beginning a very long time in youngsters and grown-ups. Bone structure does not change when individual is completely developed. Maturing in grown-up is portrayed by wrinkles, retrusion, listing skin, eye slops and so on [14-15]. Shape change because of development is introduced by Thompson [16] while confront anthropometric profile for portraying human face certain qualities is exhibited by Farank and Farank and Munro [1718]. Age invariance confront acknowledgment (AIFR) has been comprehensively arranged into three classes: I) generative ii) discriminative and iii) Deep learning. Generative technique depends on age movement strategies to change test picture to a similar age that of display picture [910]. Discriminative strategies handle acknowledgment without age movement; rather, they depend on nearby component descriptors. Discriminative learning strategies are additionally created for highlight coordinating in AIFR. As of late convolutional neural systems $(\mathrm{CNN})$ have risen as a ground-breaking machine learning model. Profound learning based AIFR strategies are treated as generative just as discriminative techniques [15].

\subsection{Generative model}

A generative model considers development of objective subject's face to be constrained by a lot of shrouded parameters. In any case, maturing procedure which should be demonstrated is exceedingly mind boggling and there are different components that influence maturing which are subject-explicit and rely upon particular age go.

The generative model has basic stages like load input picture and performs ordinary scientific task on picture. It has less yield status in contrast with discriminative model [5, 7]. Foremost part, all encompassing strategies use to create confront maturing models and construct maturing capacities to mimic or make up for maturing procedure. A functioning appearance demonstrates (AAM) is utilized to examine age estimation issues. In this method, after AAM parameters are removed from face picture a maturing capacity is constructed utilizing Genetic Algorithms to improve maturing capacity. Probabilistic maturing model is separately setup by utilizing Gaussian blend models (GMMs). In chart calculation, component purposes of a picture and their descriptors are attempted as vertices and names correspondingly. Purpose behind low execution of generative model contrasted with proposed discriminative model is programmed milestone purpose of finder that is utilized for generative model $[5,7]$.

These face maturing databases are generally gathered from checked pictures in various stances, brightening and appearance and are inadequate to get best fit outcomes. So as to have a correct model to speak to maturing procedure, one ought to utilize an immense number of preparing pictures to at present constrained face maturing databases. Legal researchers demonstrated that human face maturing firmly relies upon ethnicity and sexual orientations. Albeit human countenances have a similar general way when maturing, every ethnic and sexual orientation bunch has particular qualities in face maturing. Along these lines, it is inadequate to expect that comparable faces age in comparative routes for every single individual $[5,7]$.

\subsection{Discriminative model}

To conquer confinements of generative model, discriminative model was proposed which removed discriminative nearby highlights that are particular for each subject. Contrasted with worldwide component based methodologies, neighborhood includes inalienably have spatial area and introduction selectivity. These properties enable nearby component portrayals to be powerful to maturing, light, and articulation varieties. Face acknowledgment calculations utilized in this model are Scale Invariant Feature Transform (SIFT), multi scale nearby twofold example (MLBP), multi include discriminant examination (MFDA) and Principal Component Analysis (PCA). Each calculation has its own preference. Contrasted with worldwide appearance highlights, neighborhood highlights have been appeared to be increasingly viable in speaking to confront pictures at differing scales and introductions and vigorous to geometric mutilations and enlightenment varieties. Neighborhood picture descriptor-based method for face portrayal are SIFT\&MLBP.MFDA is an expansion and enhancement of LDA utilizing various highlights joined with two distinctive arbitrary testing strategies in highlight and test space. By arbitrary inspecting preparation set just as element space, 
different LDA-based classifiers are built and after that joined to create a strong choice by means of a combination rule [7]

\subsubsection{Densely sampled local feature description}

The entire face picture is partitioned into a lot of covering patches and after that chose neighborhood picture descriptors is connected to each fix. Removed highlights from these patches are connected together to frame a component vector with huge dimensionality for further investigation. SIFT include descriptor quantizes both spatial area and introduction of picture angle inside a s-size estimated picture fix, and processes a histogram in which each canister compares to a blend of explicit spatial area and inclination introduction. Collection of histogram containers are weighted by angle greatness and a Gaussian rot work. Filter highlight portrayal comprises of two fundamental parts: key point extraction, and highlight descriptors. Thickly test SIFT include descriptors from whole facial picture rather than just at a generally modest number of extricated key focuses [7].

\subsubsection{Multi-feature discriminant analysis (MFDA)}

The MFDA is utilized explicitly to deal with numerous capabilities with substantial dimensionality and with various scales and estimations. There are two sorts of nearby highlights (SIFT\&MLBP), each with two diverse capabilities comparing to two distinctive fix sizes. To successfully deal with these expansive quantities of highlights for improved execution, two issues ought to be survived: 1) distinctive incongruence in scale and estimation and 2) over fitting issue. MFDA calculation isn't produced just to take care of customary dimensionality decrease issue. In MFDA, various types of highlights are broken into cuts and afterward scaled by PCA standardization, and over fitting issue is understood by arbitrary examining.

The utilization of sacking system in MFDA contrasts from conventional irregular examining based models. Rather than utilizing sacking to haphazardly test information inside each class or arbitrarily chose a subset of classes, MFDA utilizes stowing to pick a subset of explicit between class test matches that are near arrangement limit for development of betweenclass dissipate framework. Along these lines, it isn't totally irregular. Purpose behind receiving this system is expansive quantities of between class test sets and not all example sets add to learning of discriminative model. Subsequently, it is practical to pick a subset of explicit between class test combines close to characterization limit as contender to build between-class dissipate grid.

By incorporating MFDA with thickly examined neighborhood include descriptors, subsequent discriminative model is appropriate for age invariant face acknowledgment issue because of accompanying reasons: (i) thickly tested nearby element portrayal conspire is both an expansion and a mix of SIFT and MLBP. In this way, it is relied upon to acquire discriminative properties of these neighborhood depiction plans, and moreover have ability in extricating age invariant highlights, for example, dispersion of edge bearing in face. (ii) MFDA has ability to adequately consolidate rich data passed on by thickly tested SIFT and MLBP descriptors, which are integral to some degree [7].

\subsection{Principal component analysis (PCA)}

PCA includes a numerical method that changes various conceivably corresonded factors into various uncorrelated factors called vital parts, identified with first factors by a symmetrical change. This change is characterized so that primary central part has as high a fluctuation as would be prudent and each succeeding segment thus has most elevated difference conceivable under limitation that it be symmetrical to first segments. PCA is delicate to overall scaling of first factors. Contingent upon field of utilization, it is likewise named discrete Karhunen- Loève change (KLT), Hotelling change or appropriate symmetrical disintegration (POD).

The real favorable position of PCA is that eigen confront approach helps decreasing span of database required for acknowledgment of a test picture. Prepared pictures are not put away as crude pictures rather they are put away as their loads which are discovered anticipating every single prepared picture to arrangement of eigen faces acquired [6].

\subsection{Deep learning vs neural networks}

Neural systems are a lot of calculations, displayed freely after human cerebrum, that are intended to perceive designs. They translate tactile information through a sort of machine observation, marking or bunching crude information. In profound learning, a convolutional neural system $(\mathrm{CNN})$ is a class of profound, feed-forward counterfeit neural systems, most generally connected to break down visual symbolism. Neural systems are utilized in numerous applications yet primary goal of neural system in face acknowledgment is possibility of preparing a framework to catch perplexing class of face designs. In neural system number of layers, quantities of hubs, learning rates, and so on are tuned widely. Neural systems are non straight in system so it is broadly utilized strategy for face acknowledgment. Thus, element extraction step might be more productive than foremost part examination. Over $90 \%$ precision in face acknowledgment processes was accomplished in literary works. Regularly, neural systems are additionally more computationally costly than customary calculations. Cutting edge profound learning calculations, which acknowledge fruitful preparing of extremely profound neural system, can take half a month to prepare totally without any preparation. Most customary machine learning calculations set aside significantly less opportunity to prepare, running from a couple of minutes to a couple of hours or days [56-57]. Multi-Layer Perceptron (MLP) with a feed forward learning calculations was presented for its straight forwardness and its capacity in regulated example coordinating. It has been effectively connected to many example order issues [58]. Research with profound learning idea is that it is class of machine learning calculation which has highlights like: i) It has many fell layers for highlight extraction. Yield of one layer fills in as contribution of second layer and ii) higher dimension highlights are gotten from lower level highlights to frame various leveled portrayal. Layers utilized in profound learning are concealed layers of counterfeit neural system. One more favorable position of profound learning is that a layer chooses best highlights. Neural systems are prepared utilizing angle back-engendering technique; heaviness of a layer is refreshed as subordinate of past layer $[59,65,66]$.

Rather than utilizing hand-created includes in machine learning's, profound learning-based techniques are pleasantly utilized in face acknowledgment. Taigman et al. [59] present a profound model utilizing convolutional neural system $(\mathrm{CNN})$ considered profound face that accomplishes close human dimension execution in face confirmation. Yi Sun et al. [60] removed profound component portrayal for face acknowledgment by utilizing mutually confront recognizable proof and check to play out a directed preparing of CNN. Various profound learning strategies have been connected to deal with face acknowledgment issues [61-64]. These days, CNNs are indicating astounding execution in AIFR field. 
Nonetheless, ponders on CNNs are as yet restricted and needs more consideration of research.

\section{COMPARATIVE ANALYSISOF AGE INVARIANTFACE RECOGNITION METHODS}

This paper discusses a critical survey of existing literatures on age invariant face recognition methods. Table 1 provides recognition rate performance of different age invariant face recognition approaches on FG-NET, MORPH, CACD and other databases. Different types of texture descriptors such as SIFT, LBP, MLBP, PCA, LDA, IFA and various modeling techniques are applied to accurately classify face images in spite of their age variations. From Table 1, it is observed that highest recognition rate provided for FGNET and Morph database are using latent factor guided convolutional neural network. Major focus in this survey is 3classesobtainable for AIFR techniques to address recognition across different perspectives. Deep learning approaches can capture diverse
Discriminative strategies rely upon age invariant facial highlights and discriminant learning systems. Discriminative strategies can possibly address major AIFR issues. Substantial dimensionality is one of constraints of nearby component descriptors in discriminative methodologies. In complex learning, nearby highlights are anticipated onto a low dimensional complex yetrecognizing a genuine age invariant complex is an intricate research issue. Profound learning AIFR techniques are fit for learning countless in nearness of maturing and different varieties and offer abnormal state of FR execution. These methodologies require huge measured databases for face portrayal and resulting learning. Because of inaccessibility of a solitary appropriate database, CNNs in AIFR are commonly prepared utilizing two separate databases, one for removing age invariant highlights and other for grouping. In this way, contrasted with different applications, CNN based AIFR techniques are when all is said in done computationally overwhelming and tedious $[15,67$, $68,69]$.

aging patterns and have also shown improvements in age estimation accuracy $[15,67,68,69]$.

Table 1: Recognition rate of age invariant face recognition methods on various databases.

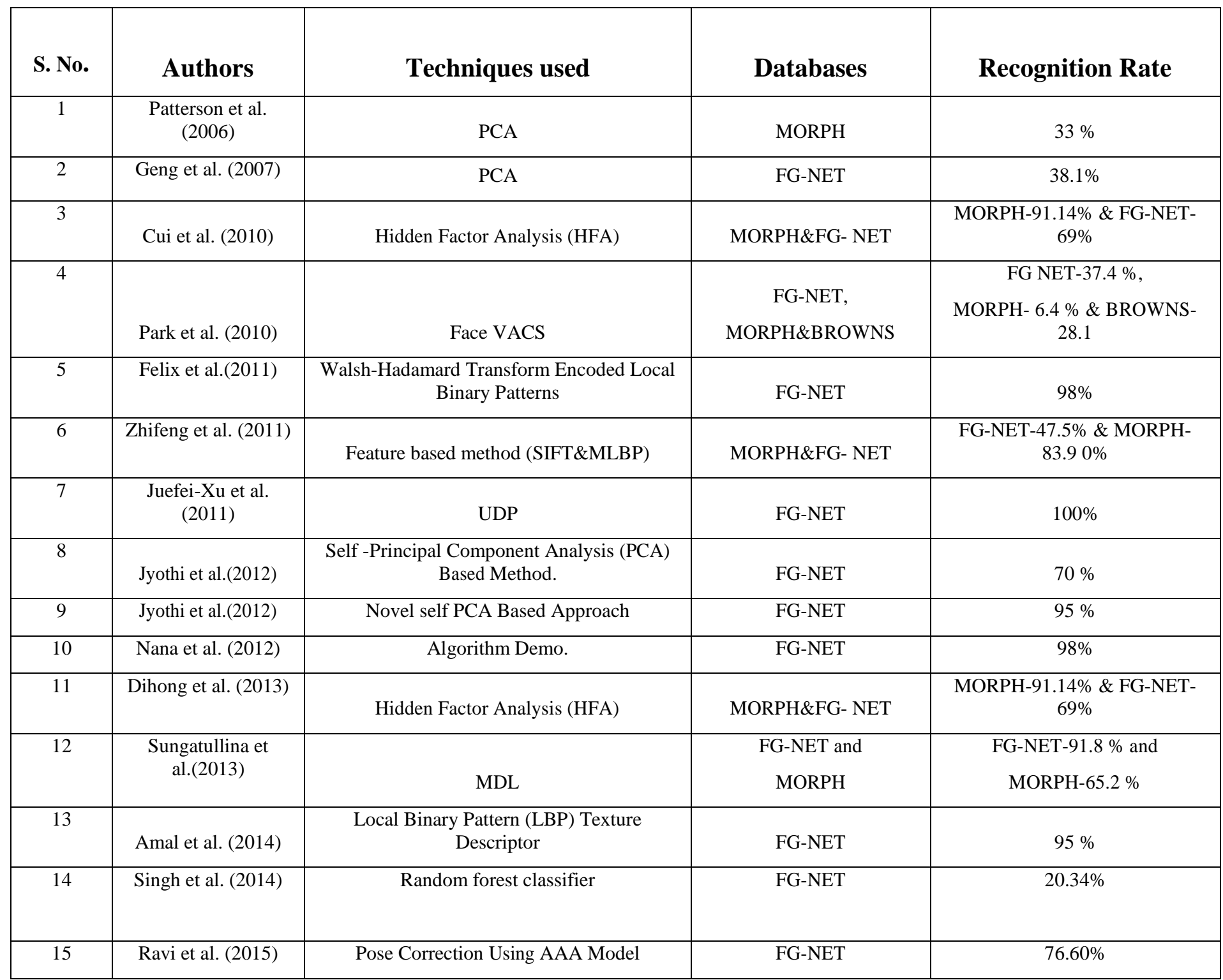




\begin{tabular}{|c|c|c|c|c|}
\hline 16 & Dihong et al. (2015) & Identity factor analysis (IFA) & MORPH, FG-NET\&LFW & $\begin{array}{l}\text { MORPH-92.6\%, FG-NET- } \\
76.2 \% \text { \& Overall- } 94.56 \%\end{array}$ \\
\hline 17 & Djamel et al. (2015) & Kernelized radial basis function technique & $\begin{array}{c}\text { Georgia Tech, } \\
\text { MORPH\&FGNET }\end{array}$ & $\begin{array}{l}\text { GeorgiaTech- } 83.6 \% \text {, Morph- } \\
83.8 \% \text { \&FG- NET-48.6\% }\end{array}$ \\
\hline 18 & $\begin{array}{l}\text { Junyong et al. } \\
\text { (2015) }\end{array}$ & PLS (Partial Least Square) model & FG-NET, MORPH & $\begin{array}{c}\text { FG NET-74.7\% \&MORPH- } \\
89.7 \%\end{array}$ \\
\hline 19 & $\begin{array}{l}\text { Xiaonan et al. } \\
\text { (2016) }\end{array}$ & PCA\&LDA & CACD\&MORPH & CACD-64\% \& MORPH-94.5\% \\
\hline 20 & Li et al. (2016) & Universal subspace analysis & MORPH Album 2 & $92.11 \%$ \\
\hline 21 & Xu et al. (2017) & Non linear factor analysis & FG-NET & $86.5 \%$ \\
\hline 24 & Li et al. (2017) & $\begin{array}{l}\text { Modified HFA\&maximum likelihood } \\
\text { approach }\end{array}$ & $\begin{array}{c}\text { FG-NET and } \\
\text { MORPH Album } 2\end{array}$ & $\begin{array}{c}\text { FG NET- } 72.8 \% \& \\
\text { MORPH Album } 2-87.94 \%\end{array}$ \\
\hline 25 & Huiling et al. (2018) & AG-IIM & $\begin{array}{c}\text { FG-NET, } \\
\text { MORPH\&CACD }\end{array}$ & $\begin{array}{c}\text { FG NET- } 88.23 \% \\
\text { MORPH- } 95.6 \% \& C A C D-89.9 \\
\%\end{array}$ \\
\hline
\end{tabular}

\section{EFFECTS OF INTRINSIC AND EXTRINSIC FACTORS ON SKIN AGEING}

Maturing is a procedure in which both inherent and extraneous determinants lead continuously to lost basic uprightness and physiological capacity Intrinsic maturing of skin happens unavoidably as a characteristic procedure of physiological changes after some time at factors hereditarily. Outward factors are changing degrees, controllable and incorporate introduction to daylight, contamination or nicotine, redundant muscle developments like squinting or grimacing, and random way of life segments, for example, diet, resting position and generally speaking wellbeing. Inborn maturing, however hereditarily decided and inalterable, isn't steady crosswise over various populaces or even unique anatomical locales on a similar person. In any case, potential parts of extraneous maturing, including nourishment, tobacco use and introduction to sun based beams, are essentially interminable, accordingly bringing about a wide scope of unmistakable indications of matured skin even inside hereditarily comparative people of a similar age [21,55].

\subsection{Intrinsic skin ageing factors}

Ethnicity: The fundamental impact of ethnicity on maturing is principally identified with contrasts in pigmentation. Abnormal amounts of pigmentation are defensive as respect to total impacts of photograph maturing; with African-Americans has minimal cutaneous contrast among uncovered and unexposed locales. African-American skin is more compacted than Caucasian skin and which may add to more protection from maturing. Wrinkling in Asians is more and with less seriousness than in Caucasians [52, 26-27, 55].

\subsubsection{Anatomical variations}

Large varieties in some skin parameters have been seen as for body. There are vast contrasts in skin thickness to body site, running from under $0.5 \mathrm{~mm}$ on eyelids to in excess of $6 \mathrm{~mm}$ on bottoms of feet. Decline in epidermal thickness with maturing is littler at sanctuary than at volar lower arm because 
of aggregate impact of photograph maturing. Skin unbending nature is a lot higher attempted than at cheek in postmenopausal ladies. Likewise, where zones of body with high blood stream, diminished with age contrasted with zones with benchmark low blood stream, no change was watched. It is regularly accepted that matured skin is characteristically less hydrated, less versatile, increasingly porous and progressively powerless to bothering [26, 28-32, 55].

\subsubsection{Hormonal changes}

The hormonal changes in skin are for most part impact of changes of estrogen levels in skin of ladies. Vulvar skin varies from majority of cutaneous epithelium skin in vulvar zone gets from three distinctive embryonic layers. Accompanying changes happen after menopause: vaginal epithelium decays, cervicovaginal discharges wind up scanty, vaginal $\mathrm{pH}$ rises, atrophic vaginitis turns out to be increasingly normal ,collagen and water content decline, pubic hair grays and ends up inadequate, labia major loses s. c. fat and furthermore labia decays. Vulvar skin is increasingly impervious to tape stripping and recuperates more rapidly in more youthful subjects than in more established ones. Furthermore, vulvar skin has an expanded rate of epidermal turnover and expanded basal cutaneous blood stream .Forearm skin is less incessant and slower response in to sodium lauryl sulfate bothering than that in more youthful skin while no age-related contrasts are found in vulvar region [22, 26, 31, 33-42, 55].

Penetrability in vulva is an unpredictable issue influenced by expanded vulvar skin hydration in nearness of hair follicles and sweat organs, cutaneous blood stream, impediment and properties of substance. Old patients are liable to contact bothering dermatitis invulvar zone by urinary dampness under impediment. Urinary smelling salts raise neighborhood $\mathrm{pH}$, which modifies obstruction working keeping skin trustworthiness and expanding danger of disease. vulvar region, in view of perspiring, impediment, vaginal release, contact, utilization of cleanliness items and incontinence, is progressively powerless to industrious vulval in maturity [26, $31,33-45,55]$.

\subsection{Extrinsic skin ageing factor Lifestyle influence}

Skin is influenced by encompassing conditions, for example, temperature and mugginess. An expansion in skin temperature may twofold evaporative water misfortune. Low temperature hardens skin and diminishes evaporative water misfortune even with a lot of moistness in air, as auxiliary proteins and lipids in skin are basically subject to temperature for suitable compliance. Prescriptions well keep skin, particularly amid hypocho-lesterolemic drugs that supply strange expanded desquamation. There are two most exogenous components; two of which correct a substantial toll on skin, are smoking and presentation to UV light [46-47, 55].

\subsubsection{Effects of smoking and nicotine}

Cigarette smoking is unequivocally related with elastosis in genders, and telangiectasia (red spots on skin) in men. Smoking causes skin harm essentially by diminishing fine blood stream to skin, which, thus, makes oxygen and supplement hardship in cutaneous tissues. Smoking increments keratinocyte dysplasia and skin unpleasantness. Smoking is a more noteworthy supporter of facial wrinkling than even sun expo-beyond any doubt. Smoking is a free hazard factor for untimely wrinkling notwithstanding when age, sun introduction and pigmentation are controlled [20, 40, $48,55]$.

\subsubsection{Exposure to UV light}

This is likewise called photograph maturing. Inherent changes happen in all skin as individual's age, including diminished turnover, substance leeway, thickness and cellularity, thermoregulation, mechanical assurance, insusceptible responsiveness, tangible recognition, sweat and sebum generation and vascular reactivity. These progressions speak to a summed up decay with couple of basic changes up to age of 50.impacts of daylight on skin are assessed to represent up to $90 \%$ of obvious skin maturing. Daylight harms skin over a range of physiological procedures [50-51].

Ultra violet radiation likewise starts harm to hereditary material. Moreover, UV radiation acts by implication to harm skin by meddling with compounds basic to DNA fix process and by obstruction with segments of insusceptible framework that demonstration to destroy cancer-causing cells. Bright light incites photochemical changes that can prompt constant impacts. Textural changes incorporate expanded harshness, straight to point keratoses and advancement of fine rhytides which advancement to more profound overlays and wrinkles. Bright band An (UVA) light enters all more profoundly. Despite fact that it doesn't cause articulated erythema, it might harm dermis more than UVB light, especially flexible tissue identified with skin maturing. Maturing strikingly expands statement of related qualities when presented to UV [26-28, $45,44-56,57]$.

\section{GUIDELINES FOR FUTURE RESEARCH AND DEVELOPMENT}

Scarcely any rules for future innovative work are recommended underneath:

1. Generative strategies are compelling fit as a fiddle displaying, while; age invariant marks are powerful in discriminative techniques. A joint methodology of worldwide and neighborhood highlights will result in better execution.

2. Profound generative models might be utilized to get familiar with a total maturing design. Joining them with profound highlights may help up execution.

3. Facial maturing is influenced by different characteristic just as outward factors, yet their quantitative examination is absent. Likewise investigation of impact of sex and race is additionally a conspicuous future bearing in improvement of a strong face acknowledgment framework.

4. Utilizing existing databases, maturing information and highlights of general regular people has not been demonstrated. Along these lines, improvement of extensive non military personnel databases with long haul development is required in not so distant future.

\section{CONCLUSIONS}

AIFR framework faces difficulties because of appearance varieties inside a subject and likenesses between subjects. Study on AIFR based approaches are sorted out in three classes: generative, discriminative and profound learning. Each methodology takes AIFR issue in an unexpected way. Generative AIFR utilize maturing models for age change while discriminative methodologies depend on age invariant highlights and learning plans. Profound learning strategies offer an incorporated structure for face portrayal and classification. However, total preparing of profound learning 
systems which require huge preparing information, utilizing little databases like FG-NET) remains a test.

Execution of AIFR framework demonstrates distinctive patterns for various ethnic starting points, guys and females. Since facial maturing relies upon different factors other than maturing, it is essential to dissect impact of characteristics and outward factors on execution. Thus, for progressively exact assessment of AIFR calculations, a facial maturing database with right statistic data and wide intra-individual varieties is need of great importance. A solitary database highlighting a vast and equivalent number of countenances per individual, over all age bunch is gravely required. Quickly, regardless of significant advance in AIFR, it is still a long way from expectations. CNN based methods are showing remarkable performance but study on CNN are still limited and needs more attention in AIFR research field. This study also covers noticeable literary works to break down and outline work done so far on age invariant face recognition and to assess them on different scales like computational speed, precision, execution consistency in inborn outward conditions on skin aging.

\section{REFERENCES}

[1] Kumar, A. V. Srivastava and P. S. Yadav, Survey on age invariant facial recognition, Advances in Computer Science and Information Technology, vol. 2, no. 5, pp. 450-453, 2015

[2] N. Ramanathan and R. Chellappa, Face verification across age progression, IEEE transactions on image processing, vol. 15 , no. 11, 2006.

[3] H. Ling, S. Soatto, N. Ramanathan and DW. Jacobs, A study of face recognition as people age, IEEE International Conference on Computer Vision, pp. 1-8, 2007.

[4] N. R. Syambas and U. H. Purwant, Image processing and ace detection analysis on face verification based on age stages, 7th IEEE, Int. Conf. on Telecommunication Systems, Services and Applications, 2012.

[5] G. Mahalingam and C. Kambhamettu, Age invariant face recognition using graph Matching, Fourth IEEE International Conference on Biometrics: Theory, Applications and Systems (BTAS), 2010

[6] S. Jyothi Nayak and M. Indiramma, Efficient face recognition with compensation for aging variations, IEEE 4th Int. Conf. on Advanced Computing, pp. 1-5, 2012.

[7] S. Jyothi Nayak, M Indiramma and N. Nagarathna , Modeling self-principal component analysis for age invariant face recognition, IEEE Int. Conf. on Computational Intelligence and Computing Research, 2012.

[8] Z. Li, U. Park, A. K. Jain., A discriminative model for age invariant face recognition, IEEE transactions on information forensics and security, vol.6, no.3, September 2011.

[9] A. Lanitis, A survey of effects of aging on biometric identity verification, International Journal of Biometrics ,vol. 2, pp. 34-52, 2009.

[10] A. Lanitis, CJ. Taylor and TF. Cootes, Toward automatic simulation of aging effects on face images, IEEE Trans Pattern Anal Mach Intell, vol. 24, pp. 442-455, 2002.
[11] N. Ramanathan, R. Chellappa and S. Biswas, Computational methods for modeling facial aging: a survey. J Vis Lang Comput, vol. 20, pp. 131-144, 2009.

[12] Y. Fu, G. Guo and TS. Huang, Age synthesis and estimation via faces: a survey, IEEE Trans Pattern Anal Mach Intell, vol. 32, pp. 1955-1976, 2010.

[13] A. Lanitis, C. Draganova and C. Christodoulou, Comparing different classifiers for automatic age estimation, IEEE Trans Syst Man Cybern B Cybern, vol 34, pp. 621-628, 2004.

[14] K. Ricanek, A. Sethuram, EK. Patterson, AM. Albert and EJ. Boone, Craniofacial aging Wiley $h$ and book of science and technology for homeland security, 2008.

[15] M. M. Sawant and K. M. Bhurch, Age invariance face recognition: a survey on facial aging databases, techniques and effect of aging, Artificial Intelligence Review, Springer, pp. 1-28, 13 October 2018.

[16] DW Thompson, On growth and form Cambridge, England, 1917

[17] L. Farkas, Anthropometry of head and face. Raven Press, New York, p XIX, 1994.

[18] L.G. Farkas and I.R. Munro, Anthropometric facial proportions in medicine. Charles C. Thomas, Springfield, 1987.

[19] N. Ramanathan, R. Chellappa and S. Biswas, Computational methods for modeling facial aging: a survey, Journal of Visual Languages and Computing, vol. 20, Issue 3, pp. 131-144, 2009.

[20] O. Friedman, Changes associated with aging face, Facial plastic surgery clinics of North America, vol. 13, Issue 3, pp. 371-380, 2005.

[21] W.F. Bergfeld, Aging skin., Int. J. Fertil. Womens Med,Vol. 42,pp. 57-66, 1997

[22] M. P. Brincat, Y.M. Baron, and R. Galea, Estrogens and skin., Climateric vol. 8, pp. 110-123, 2005.

[23] M.K. Robinson, Population differences in skin structure and physiology and susceptibility to irritant and allergic contact dermatitis: implications for skin safety testing and risk assessment, Contact Derm. vol. 41, pp. 65-79, 1999.

[24] J.L. Rees, Genetics of sun sensitivity in humans, Am. J. Hum. Genet.vol. 75, pp. 739-751, 2004.

[25] B.A. Gilchrest, A review of skin aging and its medical therapy, Br. J. Dermatol. vol. 135, pp. 867-875, 1996.

[26] F. Martini, Fundamentals of anatomy and physiology, Benjamin-Cummings, San Francisco, 2004.

[27] K. M. Sudel, K. Venzke, Mielke, H. et al, Novel aspects of intrinsic and extrinsic aging of human skin: beneficial effects of soy extract, Photochem Photobiol, vol. 81, pp. $581-587,2005$

[28] P.M. Elias, Stratum corneum architecture, metabolic activity and interactivity with subjacent cell layers, Exp. Dermatol. vol. 5, pp. 191-201 1996.

[29] M. Gray, Preventing and managing perinea dermatitis: a shared goal for wound and continence care, J. Wound Ostomy. Continence Nurs. vol. 31, pp. S2-S9, 2004. 
[30] S. Neerken, G.W. Lucassen, M.A. Bisschop, E. Lenderink, and T. A. Nuijs, Characterization of age-related effects in human skin: A comparative study that applies confocal laser scanning microscopy and optical coherence tomography, J. Biomed. Opt. vol 9, pp. 274$281,2004$.

[31] M. Farage, and Maibach, H. (eds), Vulva: Anatomy, Physiology and Pathology. Informal Healthcare, New York, pp.27-42, 2006.

[32] J. M. Waller and H.I. Maibach, Age and skin structure and function, a quantitative approach (I): blood flow, $\mathrm{pH}$, thickness, and ultrasound echo-genicity, Skin Res. Technol. vol. 11, pp. 221-235, 2005.

[33] G. Hall and T. J. Phillips, Estrogen and skin: effects of estrogen, menopause, and hormone replacement therapy on skin, J. Am. Acad. Dermatol, vol. 53, pp. 555-568, 2005.

[34] V. S. vrain, B.F and G. B , Biology of estrogens in skin: implications for skin aging, Exp. Dermatol, vol. 15, pp. 83-94, 2006.

[35] G. K. Hall, andT. J. Phillips, Skin and hormone therapy, Clin.Obstet. Gynecol, vol. 47, pp. 437-449, 2004.

[36] N. J. Raine, Fenning, M.P. Brincat, and Y. MuscatBaron, Skin aging and menopause implications for treatment, Am. J. Clin. Dermatol, vol. 4, pp. 371-378, 2003.

[37] M. J. Thornton, Biological actions of estrogens on skin, Exp. Dermatol, vol. 11, pp. 487-502, 2002.

[38] M. A. Farage, K.W. Miller, P. Elsner, and H. I. Maibach, Structural characteristics of aging skin: a review, J. Toxicol. Cutaneous Ocul.Toxicol, vol. 26, pp. $1-15,2001$

[39] M. A. Farage, K.W. Miller, P. Elsner and H. I. Maibach, Functional and physiological characteristics of aging skin: a review, Aging clinical and experimental research, vol. 20, Issue 3, pp. 62-71, 2008.

[40] C. Castelo-Branco, F. Figueras, M. J. Martinez de Osaba, and J.A. Varnell, Facial wrinkling in postmeno-pausal women, Effects of smoking status and hormone replacement therapy, Maturitas, vol. 29, pp. 75-86, 1998.

[41] D. Wilhelm, P. Elsner, and H. I. Maibach, Standardized trauma (tape stripping) in human vulvar and forearm skin. Effects on transepidermal water loss, capacitance and $\mathrm{pH}$, ActaDerm.Venereol, vol. 71, pp. 123-126, 1991.

[42] P. Elsner, D. Wilhelm and H.I. Maibach, Sodium lauryl sulfate-induced irritant contact dermatitis in vulvar and forearm skin of premenopausal and post-menopausal women, J. Am. Acad. Dermatol, vol. 23, pp. 648-652, 1990.

[43] R. G. Glogau, Physiologic and structural changes associated with aging skin, Dermatol. Clin, vol. 15, pp. 555- 559, 1997.

[44] P. Marren, F. Wojnarowska and S. Powell, Allergic contact dermatitis and vulvar dermatoses, Br. J. Dermatol, vol. 126, pp. 52-56, 1992.

[45] N. A. Fenske and C.W. Lober, Structural and functional changes of normal aging skin, J. Amer. Acad. Dermatol, vol. 15 , pp. 571-585, 1986.
[46] S. M. Jackson, M. L. Williams, K. R. Feingold, and P.M. Elias, Pathobiology of stratum corneum, West. J. Med., vol. 158, pp. 279-285, 1993.

[47] C. Kennedy, M. T. Bastiaens, C. D. Bajdik, et al., Leiden Skin Cancer Study. Effect of smoking and sun on aging skin., J. Invest. Dermatol. vol. 120, pp. 548-554, 2003.

[48] Y. H. Leow, and H. I. Maibach, Cigarette smoking, cutaneous vasculature, and tissue oxygen, Clin.Dermatol., vol. 16, pp. 579-584, 1998.

[49] [49] WebRef.org. [WEBSITE]. Available at: http:// www.webref.org/cancer/p/pack_year.htm, accessed on 2/27/06. Inverson Software Co.

[50] C. O. Barl and, E. Zettersten, B.S. Brown, J. Ye, P. M. Elias and R. Ghadially, Imiquimod-induced interleukin-1 alpha stimulation improves barrier homeostat is in aged murin epidermis, J. Invest. Derma-tol. vol. 122, pp. 330336, 2004.

[51] S. Kang, J. H. Chung, J. H. Lee, G.J. Fisher, Y.S. Wan, E.A. Duell and J. J. Voorhees, Topical N-acetyl cysteine and genistein prevent ultraviolet-light-induced signaling that leads to photo aging in human skin in vivo, J. Invest. Dermatol, vol.120, pp. 835-841, 2003.

[52] K. Sauermann, S. Jaspers, U. Koop and H. Wenck, Topically applied vitamin $\mathrm{C}$ increases density of dermal papillae in aged human skin, BMC Dermatol. vol. 4, no.13, 2004.

[53] S Seite, C. Bredoux, , D. Compan, et al., Histological evaluation of a topically applied retinol-vitamin C combination. Skin, Pharmacol. Physiol. vol. 18, pp. $81-$ 87,2005

[54] A. Sesto, M. Navarro, F. Burslem, and J. L. Jorcano, Analysis of ultraviolet B response in primary human keratinocytes using oligonucleotide micro-arrays, Proc. Natl Acad. Sci. U.S.A. vol. 99, pp. 2965-2970, 2002.

[55] M. A. Farage, K. W. Miller, P. Elsne and H. I. Maibach, Intrinsic and extrinsic factors in skin ageing: a review, International Journal of Cosmetic Science, vol. 30, pp 87-95, 2008

[56] Ming-Hsuan Yang, D. J. Kriegman and N. Ahuja, Detecting faces in images: A survey, IEEE Trans on Pattern Analysis and Machine Intelligence, vol. 24, no. 1, January 2002

[57] M. Kirby and L. Sirovich, Application of KarhunenLoeve procedure for characterization of human faces. IEEE Trans. Patt. Anal. Mach. Intell., vol. 12, no. 1, pp. 103-108, 1990

[58] X. Li and S Areibi, A hardware/software code sign approach for face recognition,16th, International Conference on Microelectronics, Tunisia, 2004.

[59] Y. Taigman, M. Yang, M. Ranzato and L. Wolf, Deep face: Closing gap to human-level performance in face verification in CVPR, pp. 1701-1708, 2014.

[60] Y. Sun, Y. Chen, X. Wang and X. Tang, Deep learning face representation by joint identification-verification in advances in neural information processing systems, $\mathrm{Z}$. Ghahramani, M. Welling, C. Cortes, N. D. Lawrence, and K. Q. Weinberger, Eds., pp. 1988-1996, 2014. 
[61] Z. Zhu, P. Luo, X. Wang and X. Tang, Deep learning identity preserving face space, in ICCV, pp. 113-120, 2013.

[62] Y. Zhang, M. Shao, E. K. Wong and Y. Fu, Random faces guided sparse many-to-one encoder for poseinvariant face recognition, in ICCV, pp. 2416-2423, 2013.

[63] M. Kan, S. Shan, H. Chang, and X. Chen, Stacked progressive auto encoders (spae) for face recognition across poses, in CVPR, pp. 1883-1890, 2014.

[64] H. Zhai, C. Liu, H. Dong, Y. Ji, Y. Guo and S. Gong, Face verification across aging based on deep convolutional networks and local binary patterns, in International Conference Intelligence Science and Big Data Engineering, pp. 341-350, 2015.

[65] Y. Naik, Detailed survey of different face recognition approaches, International Journal of Computer Science and Mobile Computing, Vol.3, Issue.5, pp. 1306-1313, May- 2014.

[66] D. Sinha, J. P. Pandey and B. Chauhan, A deep learning approach for age invariance face recognition, International Journal of Pure and applied Mathematics, vol. 117, no. 21, pp. 371-389, 2017

[67] Y. Wang, D. Gong, Z. Zhou, X. Ji, H. Wang, Z. L, W. Liu, T. Zhang, Orthogonal deep features decomposition for age-invariant face recognition, European Conference on Computer Vision, pp. 764-779, 2018.

[68] H. Zhou, K. M. Lam, Age-invariant face recognition based on identity inference from appearance age, Pattern recognition, vol. 76, pp. 191-202, 2018.

[69] W. Yitong, G. Dihong, Z. Zheng, J. Xing, W. Hao, Li Zhifeng, L Wei, and Z Tong, Orthogonal deep features decomposition for age-invariant face recognition, IEEE, Pattern Anal Mach Intell, vol. 29, pp. 2234-2240, 2018. 